\title{
Resistance to nitroprusside neurotoxicity in perinatal rat brain
}

\author{
William F. Maragos ${ }^{\mathrm{a}}$, Faye S. Silverstein ${ }^{\mathrm{a}, \mathrm{b}}$ \\ Departments of ${ }^{a}$ Neurology and ${ }^{b}$ Pediatrics, University of Michigan, Ann Arbor, MI, USA
}

Received 10 January 1994; Revised version received 8 March 1994; Accepted 9 March 1994

\begin{abstract}
Nitric oxide (NO) may mediate some of the toxic effects of the excitatory amino acid (EAA) glutamate when there is overactivation of the $N$-methyl-D-aspartate (NMDA) receptor. In the developing rodent nervous system, NMDA neurotoxicity peaks at postnatal day 7. To assess whether NO toxicity exhibits a similar developmental profile, we injected the NO generator sodium nitroprusside into the immature and adult rodent hippocampal formation and striatum, using a dose known to damage the adult nervous system. Contrary to our expectations, we found the immature brain highly resistant to the toxic effects of sodium nitroprusside.
\end{abstract}

Key words: Nitric oxide; Nitroprusside; Neurotoxicity; Free Radical; Perinatal; Rodent

A recent report demonstrated that intracerebral injections of sodium nitroprusside, a compound that generates nitric oxide (NO), produce focal necrotic lesions in the adult rat hippocampus [19]. Evaluation of nitroprusside neurotoxicity was prompted by a growing body of evidence that NO has intrinsic neurotoxic properties and mediates the neurotoxic effects of the excitatory amino acid (EAA) neurotransmitter glutamate. NO is generated by the catalysis of arginine to citrulline by nitric oxide synthase (NOS) $[17,20]$; in neurons, NOS is a calmodulin-dependent enzyme [2] which is activated by calcium influx following $N$-methyl-D-aspartate (NMDA) receptor stimulation [2,9]. Excessive NMDA receptor activation, by increased synaptic concentrations of endogenous glutamate or by experimentally administered NMDA agonists, initiates a cascade of events resulting in cell death [5]. Evidence that NO generation contributes to EAA neurotoxicity is provided by the observation that chelators of NO prevent glutamate-induced cell death in rat cortical cultures [6], and by reports that inhibitors of NOS prevent EAA-induced neuronal damage both in vitro $[6,14]$ and in vivo $[8]$ and in some

\footnotetext{
* Corresponding author. Address: University of Michigan Medical Center, Room 6028 Kresge II, Ann Arbor, MI 48109-0570, USA. Fax: (1) (313) 7644279
}

models of cerebral hypoxic-ischemic injury [3,15,22,23]. In certain experimental paradigms, these same inhibitors may limit the neurotoxicity of the NMDA agonist quinolinic acid in vivo [28], but paradoxical enhancement of toxicity has also been reported [10].

Considerable experimental data indicate that developmental stage influences CNS susceptibility to EAA agonist neurotoxicity. For example, although the immature rodent brain is resistant to kainate [4], it is highly vulnerable to NMDA toxicity; susceptibility to NMDA-mediated forebrain injury peaks at postnatal day 7 (P7) [21]. Whether NO itself is toxic in the developing nervous system is unknown. If NO mediates NMDA-induced brain injury, then it might be expected that the developing brain would be particularly susceptible to nitroprusside neurotoxicity. To test this hypothesis, we performed direct intrahippocampal and intrastriatal injections of sodium nitroprusside in $\mathrm{P} 7$ and adult rats, and assessed the severity of resulting brain injury in animals sacrificed 5 days later.

Eleven unsexed P7 rats ( $8-12 \mathrm{~g}$ ) and 10 adult male rats (175-200 g; Sprague-Dawley, Harlan) were used. All animal use procedures were in accordance with the NIH Guide for the Care and Use of Laboratory Animals and were approved by the University of Michigan Committee on the Use and Care of Animals. Sodium nitroprusside was obtained from Sigma (St Louis, MO).

Ether anesthesia was used for both perinatal and adult 
rat surgery. Solutions of nitroprusside (dissolved in room-temperature sodium phosphate buffer, $\mathrm{pH}$ 7.4) were prepared immediately prior to surgery to minimize exposure to light. Using a 30-gauge cannula, seven P7 rats (three and four animals/experiment, in two independent experiments using different batches of nitroprusside) and four adult rats each received $1-\mu 1$ injections of 33 nmol sodium nitroprusside into the CA3 subfield of the right hippocampal formation; four $\mathrm{P} 7$ and six adult animals received injections of the same volume of sodium nitroprusside in the right striatum. The anesthetized P7 animals were positioned with bregma and lambda in the same horizontal plane and stereotaxic coordinates for the striatum, relative to bregma, were AP $-0.2 \mathrm{~mm}$, ML $2.5 \mathrm{~mm}$, DV $3.5 \mathrm{~mm}$; for CA3, coordinates were: $\mathrm{AP}-1.7 \mathrm{~mm}, \mathrm{ML} 2.7 \mathrm{~mm}$, and $\mathrm{DV} 2.7 \mathrm{~mm}$. In the adults, with the incisor bar at $-2.3 \mathrm{~mm}, \mathrm{CA} 3$ coordinates were, relative to bregma $\mathrm{AP}-4.0 \mathrm{~mm}, \mathrm{ML}-3.0 \mathrm{~mm}$ and DV $3.6 \mathrm{~mm}$ from the cortical surface; the striatum was targeted $\mathrm{AP}+1.0 \mathrm{~mm}$ and $\mathrm{ML} 2.5 \mathrm{~mm}$ from bregma and $5.0 \mathrm{~mm}$ ventral from the surface of the brain. Following injections, the cannula was left in place for one minute. Two of the adult rats died during surgery. Controls receiving intraparenchymal injections of vehicle were not included in this study since, in previous experiments using our injection methods, phosphate buffer produced only subtle well-circumscribed lesions adjacent to the needle tract in both the immature and adult brain. Animals were sacrificed 5 days later by decapitation, the brains quickly removed and frozen on powdered dry ice. The brains were stored at $-70^{\circ} \mathrm{C}$ until they were sectioned.

For histological evaluation, $20-\mu \mathrm{m}$ frozen coronal sections obtained at $80-\mu \mathrm{m}$ intervals were thaw-mounted on gelatin-coated glass slides. Following a 2-day exposure to paraformaldehyde vapor at room temperature, sections were stained for Nissl substance using Cresyl violet.
Assessment of the lesions was made using a dissecting stereoscope.

In brains of adult rats that had received striatal injections of sodium nitroprusside there were discolored areas of necrosis, $\sim 3$ by $3 \mathrm{~mm}$, on the ventral surface of the brain in the anterior temporal region. These regions represent the most ventral extent of the lesion. In brains of animals that received hippocampal injections, slight depressions of the overlying cortical surface were evident. Gross inspection of the immature rat brains following injections into either $\mathrm{CA} 3$ or the striatum revealed no obvious abnormalities.

Injections of sodium nitroprusside into adult CA3 resulted in marked atrophy of the entire hippocampus with loss of both pyramidal and granule cells (Fig. 1 A). In all cases, there was evidence of variable degrees of neuronal necrosis in the adjacent thalamus and the overlying cortex. Of particular interest was the sparing of the corpus callosum in those sections in which there were both hippocampal and cortical lesions. Injections of nitroprusside were also destructive when applied to the striatum (Fig. 1B). These lesions typically involved approximately one half or greater of the striatum at the center of the lesion and extended through its entire rostrocaudal extent. Some of the lesions also extended ventrally into nucleus accumbens and into cortical regions laterally. The corpus callosum was again spared and appeared to limit the extent of injury.

In contrast with the extensive abnormalities in the adult, in the younger animals no obvious lesions were produced by sodium nitroprusside injection into either the hippocampus or striatum (Fig. 2). In five of the seven animals that had received injections in the hippocampus, minimal mechanical damage was noted around the cannula tract. Features of mechanical injury ranged from small necrotic cysts (Fig. 2A, asterisk) along or at the tip of the needle tract to focal disruption of the CAl pyram-
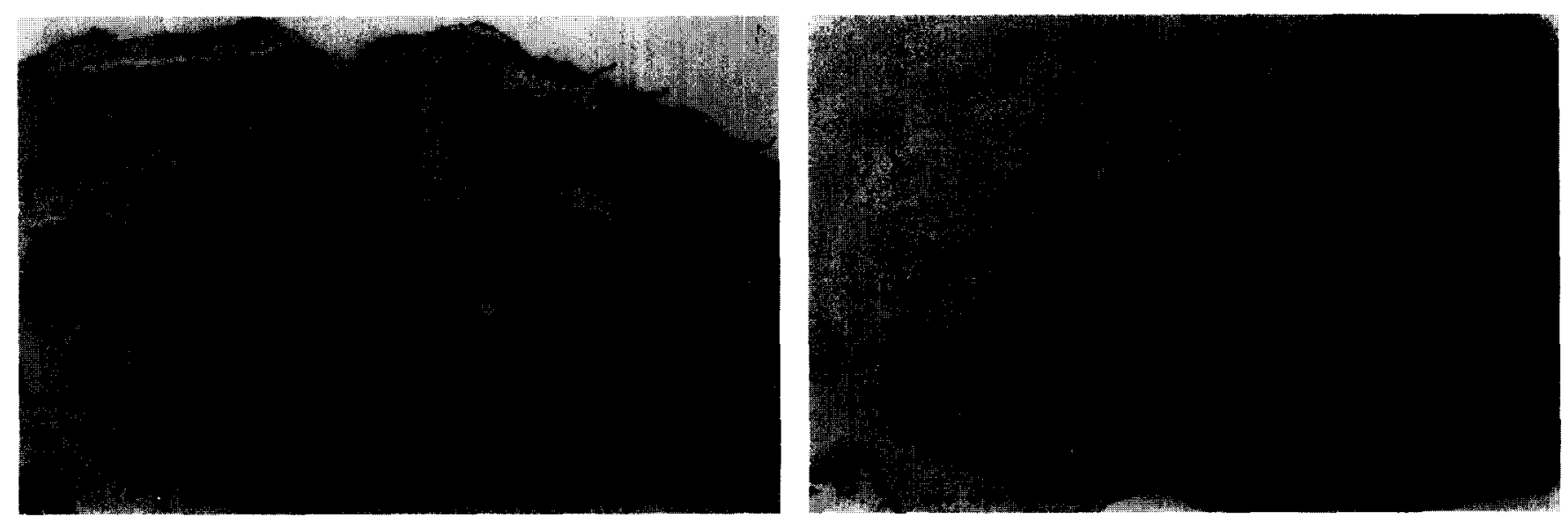

Fig. 1. Photomicrographs of Cresyl violet-stained sections of the adult rat at the level of the hipppocampal formation (A) and the striatum (B) 5 days following injection of $33 \mathrm{mmol}$ nitroprusside. Both lesions are characterized by marked neuronal loss and possess regions of liquefaction. Note the involvement of the cortex overlying the hippocampus and the sparing of the corpus callosum. cc; corpus callosum, ST: striatum. 

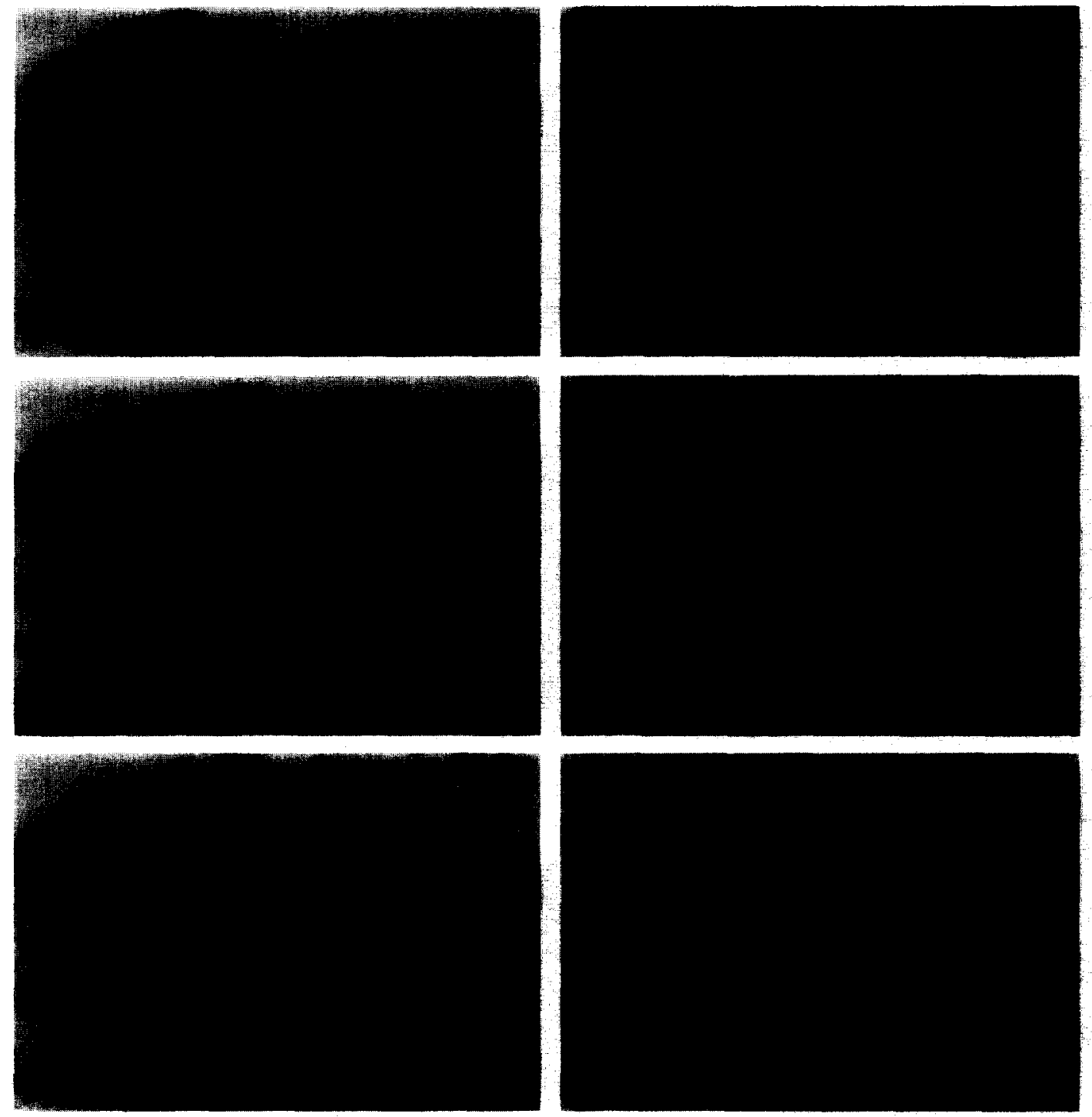

Fig. 2. Photomicrographs of Cresyl violet-stained sections taken from six individual P12 rat brains at the level of the hippocampal formation (A-C) and the striatum (D-F) 5 days following injections of $33 \mathrm{nmol}$ nitroprusside on P7. In the hippocampus, needle penetration in five of the seven animals resulted in mechanical damage ranging from necrotic cysts (A, asterisk) to a gap in the pyramidal cell layer of CAl (B, arrowhead). In two of the seven animals, focal cell loss in CA3 was observed (as illustrated in panels $\mathrm{A}$ and $\mathrm{C}$. arrowheads). The most severe lesion elicited by striatal injections (illustrated in panel D) was a narrow linear region of tissue disruption immediately adjacent to the lateral ventricle. Needle tracts, without evidence of destruction, can be identified exiting the corpus callosum in both $\mathrm{E}$ and $\mathrm{F}$.

idal cell layer by the needle (Fig. 2B, arrowhead). In four of these five animals, there was no other evidence of injury. In two animals (including one with a mechanical lesion and one in which no tract was evident), there were subtle discrete lesions in the pyramidal cell layer consisting of focal cell loss without gliosis (Fig. 2A,C, arrowheads). In one of seven, neither the cannula tract nor focal cell loss was detected. In one of the four animals that had received an injection into the striatum, the mechanical damage by the cannula resulted in a narrow region of disrupted striatal morphology adjacent to the lateral ventricle (Fig. 2D, arrowheads); in two of the four animals, the cannula tract was identified exiting the cor- pus callosum (Fig. 2B,C), and in one brain (not shown) there was no discernible evidence of injury.

These results demonstrate that direct intracerebral injection of sodium nitroprusside is not neurotoxic in the developing rat brain. It is unlikely that our findings can be explained by failure of the immature brain to generate NO since it has been shown that nitroprusside stimulates the formation of NO in slices of neonatal cerebellum [26] and that the NMDA agonist quinolinate [28] inereases striatal production of NO in P7 rats. Several mechanisms could account for this significant developmental difference in susceptibility to nitroprusside neurotoxicity. Following its liberation, NO reacts with endogenous super- 
oxide anions to form peroxynitrite, a highly reactive intermediate which is thought to mediate NO neurotoxicity [1]. The immature brain, which has a lower rate of oxidative metabolism than the adult [7], may generate fewer superoxide free radicals and, thus, may subsequently form non-toxic amounts of peroxynitrite when NO is generated from nitroprusside.

Another possible explanation for the observed difference in effects of nitroprusside in the developing and adult brain may relate to the redox state of NO itself [18]. Sodium nitroprusside has strong nitrosonium ion $\left(\mathrm{NO}^{+}\right)$ properties which require chemical reduction before NO is generated. This can be accomplished by reaction with endogenous thiols which form complexes, such as those in proteins, that ultimately release NO. Since the redox milieu in the adult and neonatal brains are likely to differ [13], it is conceivable that the adult brain favors the formation of NO while the immature brain favors the less toxic $\mathrm{NO}^{+}$species. It is also important to note that we are not yet able to rule out a contribution from the ferrocyanide portion of the nitroprusside molecule which has recently been demonstrated to attenuate NMDA stimulated calcium influx in cerebellar granule cells [16], and may have differential effects on NMDA receptors expressed in neonatal and adult brain.

The findings reported herein are somewhat discrepant with the results of two groups who independently demonstrated that pretreatment with the NOS inhibitor $\mathrm{L}-N^{\mathrm{G}}$-nitroarginine (NO-Arg) was neuroprotective in a focal stroke model in the 7-day-old rat $[11,27]$. This finding suggests that endogenous NO production at this developmental stage was a mediator of ischemic neuronal injury. It is conceivable that hypoxia/ischemia alters the intracellular milieu in such a way as to augment NO toxicity, e.g., by increasing production of superoxide anions, which, coupled with hypoxia/ischemia induced NMD $\Lambda$ receptor activation and subsequent NO release, would favor the production of the peroxynitrite intermediate. Alternatively, the neuroprotective effects of an NOS inhibitor may have been attributable to inhibition of non-neuronal NO production by endothelial cells or glia, or to induced hypothermia which itself is neuroprotective.

The observation that developmental stage influences susceptibility to nitroprusside neurotoxicity may help explain some of the discrepancies in results of studies on the effects of NOS inhibitors on glutamate-induced toxicity in cortical cultures [12,24,25]. In these and other culture systems, cells are harvested from embryonic brain tissue in which the NO transduction pathway may not be fully functional, and the extent of maturation that occurs in vitro may vary. Understanding the mechanisms that underlie these developmental differences may help elucidate the contribution of NO to excitotoxic neuronal injury and aid in development of new pharmacologic strategies to attenuate EAA-mediated neurotoxicity.
Supported by grants (to F.S. Silverstein) from the American Heart Association (92-845) and USPHS (NS 26142)

[1] Beckman, J.S., Beckman, T.W., Chen, J., Marshall, P.A. and Freeman. B.A., Apparent hydroxyl radical production by peroxynitrite: implications for endothelial injury from nitric oxide and superoxide, Proc. Natl. Acad. Sci. USA, 87 (1990) 1620-1624.

[2] Bredt, D.S. and Snyder, S.H., Isolation of nitric oxide synethase, a calmodulin-requiring enzyme. Proc. Natl. Acad. Sci. USA. 87 (1990) 682-685.

[3] Buisson, A., Plotkine, M. and Boulu, R.G., The neuroprotective effect of a nitric oxide inhibitor in a rat model of focal cerebral ischaemia, Br. J. Pharmacol., 106 (1992) 766-767.

[4] Campochiaro, P. and Coyle, J.T., Ontogenetic development of kainate neurotoxicity correlates with glutamatergic innervation, Proc. Natl. Acad. Sci. USA. 75 (1978) 20252029.

[5] Choi, D.W., Excitoloxic cell death, J. Neurobiol., 23 (1992) 126 I 1276.

[6] Dawson, V.L., Dawson. T.M., London, E.D., Bredt, D.S. and Snyder, S.H., Nitric oxide mediates glutamate neurotoxicity in primary cortical cultures, Proc. Natl. Acad. Sci. USA. 88 (1991) 6368-6371.

[7] Duffy, T.E., Kohle, S.J. and Vannucci, R.C., Carbohydrate and energy metabolism in perinatal rat brain: relation to survival in anoxia, J. Neurochem., 24 (1975) 271--276.

[8] Fujisawa, H., Dawson, D., Browne, S.E., Mackay, K.B., Bullock, R. and McCulloch, J., Pharmacological modification of glutamate neurotoxicity in vivo, Brain Res., 629 (1993) 73-78.

[9] Garthwaite, J., Garthwaite, G., Palmer, R.M.J. and Moncada, S., NMDA receptor activation induces nitric oxide synthesis from arginine in brain slices, Eur. J. Pharmacol., 172 (1989) 413-416.

[10] Haberny, K.A., Pou, S. and Eccles, C.U., Potentiation of quinoli nate-induced hippocampal lesions by inhibtion of NO synthesis, Neurosci. Lett., 146 (1992) 187-190.

[11] Hamada, Y., Hayakawa, T., Hattori, H. and Mikawa, H., Inhibitor of nitric oxide synthesis reduces hypoxic-ischemic brain damage in the neonatal rat. Pediatr. Res., 35 (1994) 10-14.

[12] Hewett. S.F., Corbett, J.A., McDaniel, M.L. and Choi, D.W., Inhibition of nitric oxide formation does not protect murine cortical cell cultures from $N$-methyl-1)-aspartate neurotoxicity, Brain Res., 625 (1993) 337-341.

[13] Hothersall. I.S., El-Hassan, A., McLean. P. and Greenbaum, A.L., Age-related changes in enzymes of rat brain, 2. Redox systems linked to NADP and glutathione. Enzyme, 26 (1981) 271 $2 / 6$

[14] Izumi, Y., Benz, A.M., Clifford, D.B. and Zorumski, C.F., Nitric oxide inhibitors attenuate $N$-methyl-D-aspartate excitotoxicity in rat hippocampal slices, Neurosci. Lett., 135 (1992) 227-230.

[15] Kader, A., Frazzini, V.I., Solomon, R.A. and Trifiletti, R.R., Nitric oxide production during focal cerebral ischemia in rats, Stroke, 24 (1993) 1709.1716.

[16] Kiedrowski, L.. Costa, E. and Wroblewski, J.T., Sodium nitroprusside inhibits $N$-methyl-D-aspartate-evoked calcium influx via a nitric oxide- and cGMP-independent mechanism, Mol. Parmacol., 41 (1992) $779-784$

[17] Knowles, R.G., Palacios, M., Palmer, R.M.J. and Moncada S., Formation of nitric oxide from L-arginine in the central nervous system: a transduction mechanism for stimulation of the soluble guanylate cyclase, Proc. Natl. Acad. Sci. USA, 86 (1989) 5159 5162 .

[18] Lipton, S.A., Choi, Y.-B., Pan, Z.-H., Lei, S.Z., Chen, H.-S.V., Sucher, N.J., Loscalzo, J., Singel, D.J. and Stamler, J.S., A redoxbased mechanism for the neuroprotective and neurodestructive effects of nitric oxide and related nitroso-compounds, Nature (London), 364 (1993) 626-632. 
[19] Loiacono, R.E. and Beart, P.M., Hippocampal lesions induced by microinjection of the nitric oxide donor nitroprusside, Eur. J. Pharmacol, 216 (1992) 331-333.

[20] Marletta, M.A., Yoon, P.S., Iyengar, R., Leaf, C.D. and Wishnok, J.S., Macrophage oxidation of l.-arginine to nitric and nitrate: nitric oxide is an intermediate, Biochemistry, 27 (1988) 8706 8711 .

[21] McDonald, J.W., Silverstein, F.S. and Johnston, M.V., Neurotoxicity of $N$-methyl-b-aspartate is markedly enhanced in developing rat central nervous system, Brain Res., 459 (1988) 200--203.

[22] Nagafuji, T., Matsui, T., Koide, T. and Asano, T., Blockade of nitric oxide formation by ${ }^{\infty}$-nitro-l-arginine mitigates ischemic brain edema and subsequent cerebral infarction if rats, Neurosci. Lett., 147 (1992) 159-162.

[23] Nowicki, J.P., Duval, D., Poignet, H. and Scatton B., Nitric oxide mediates neuronal death after focal cerebral ischemia in the mouse, Eur. J. Pharmacol., 204 (1991) 339-340.
[24] Pauwels P.J. and Leysen, J.E., Blockade of nitric oxide formation does not prevent glutamate-induced neurotoxicity in neuronal cul. tures from rat hippocampus, Neurosci. Lett., 143 (1992) 2730.

[25] Regan, R.F., Renn, K.E. and Panter, S.S., NMDA neurotoxicity in murine cortical cell cultures is not attenuated by hemoglobin or inhibition of nitric oxide synthesis, Neurosci. Lett., 153 (1993) $53-56$.

[26] Southam, E., East, S.J. and Garthwaite, J., Excitatory amino acid receptors coupled to the nitric oxide/cyclic GMP pathway in rat cerebellum during development, J. Neurochem., 56 (1991) 2072 2081 .

[27] Trifiletti, R.R., Neuroprotective effects of $\mathrm{N}^{\mathrm{G}}$-nitro-L-arginine in focal stroke in the 7-day old rat, Eur. J. Pharmacol.. 218 (1992) 197-198.

[28] Trifiletti, R.R., Macaya, A. and Bolan, E.A., Nitric oxide mediates quinolinate neurotoxicity in the rat pup, Ann. Neurol., 34 (1993) 444. 